\title{
Paramétrage, utilisation et apport de l'analyse cepstrale en maintenance prévisionnelle
}

\author{
Olivier Cousinard ${ }^{1, a}$, Philippe Rousseau ${ }^{2}$, Fabrice Bolaers et Patrick Marconnet ${ }^{2}$ \\ 1 SPIM INDUSTRIES, ZA La Neuvillette, rue Rosa Luxemburg, 51100 Reims, France \\ 2 Groupe de Mécanique, Matériaux et Structures, Université de Reims Champagne-Ardenne, IUT de Reims, rue des Crayères, \\ BP 1035, 51687 Reims Cedex 2, France
}

Reçu le 4 décembre 2002, accepté le 6 février 2004

\begin{abstract}
Résumé - Cet article propose une évaluation de l'analyse cepstrale dans le suivi vibratoire des machines tournantes afin de mettre en œuvre une maintenance prévisionnelle. Après avoir rappelé les propriétés du cepstre pour un diagnostic vibratoire, nous précisons les valeurs de paramétrage des mesures, afin d'effectuer une analyse efficace. Nos travaux sont validés par deux applications complémentaires réalisées en milieu industriel : l'une sur un multiplicateur tournant à grande vitesse, l'autre sur un réducteur tournant à très faible vitesse. Le défaut recherché est un écaillage de denture, mais la comparaison suivant deux directions de mesurage des signaux des accéléromètres a également permis dans la deuxième application de détecter un autre type d'avarie.
\end{abstract}

Mots clés : Suivi de l'endommagement / maintenance prévisionnelle / analyse vibratoire / machines tournantes

\begin{abstract}
Parametrising use and cepstral analysis contribution in forward-looking maintenance. This article suggests a cepstral analysis for swivel machines vibratory follow-up, to make use of a forward-looking maintenance. Further to the remind of cepstrum properties for vibratory diagnosis, we clarify measures parametrizing values, to make a good analysis. Our works are authenticated with two complementary applications realised in industrial circles: the first with a multiplier running at maximum revs, the other with a speed reducer ticking over. The defect, we try to detect, is a chipping cog, but the comparison with two accelerometers signals measure directions has also permitted to detect an other type of damage, in the second application.
\end{abstract}

Key words: Damaging follow-up / forward-looking maintenance / vibratory analysis / swivel machines

\section{L'analyse cepstrale}

\subsection{Introduction}

Les objectifs principaux de la maintenance prévisionnelle sont la détection d'une anomalie sur une machine avant qu'elle n'entraîne une grave défaillance, et le suivi de l'état d'usure ou de détérioration des machines composants un système de production, grâce à des indicateurs significatifs, de manière à planifier d'éventuelles interventions. Il apparaît donc dans ce dernier cas une notion essentielle de prévision des événements qui explique l'intérêt constant que les industriels et les laboratoires portent à ce domaine.

Le diagnostic dans la majorité des cas est établi en interprétant les signaux vibratoires émis par les machines

\footnotetext{
a Auteur correspondant : o.cousinard@altead.com
}

en fonctionnement. Plusieurs techniques de traitement de ces signaux se sont développées dont l'analyse spectrale apparaît dans la majorité des cas, comme la plus efficace. Néanmoins, elle présente des limites et des techniques complémentaires telles que les analyses du signal d'enveloppe, temps-fréquence ou cepstrale prennent alors le relais afin d'affiner ou de sécuriser le diagnostic.

L'objet de cette étude est précisément de présenter un outil complémentaire, l'analyse cepstrale, qui apporte des informations plus fines notamment lors de la détection de chocs dans les systèmes à engrenages, très répandus dans l'industrie. Elle se décompose en deux parties.

Dans la première, après avoir rappelé les principaux défauts rencontrés sur des réducteurs à engrenages, ainsi que les principaux aspects théoriques du spectre, nous précisons son domaine d'utilisation et nous proposons des tables de paramétrage indispensables à une bonne exploitation de cet outil. 


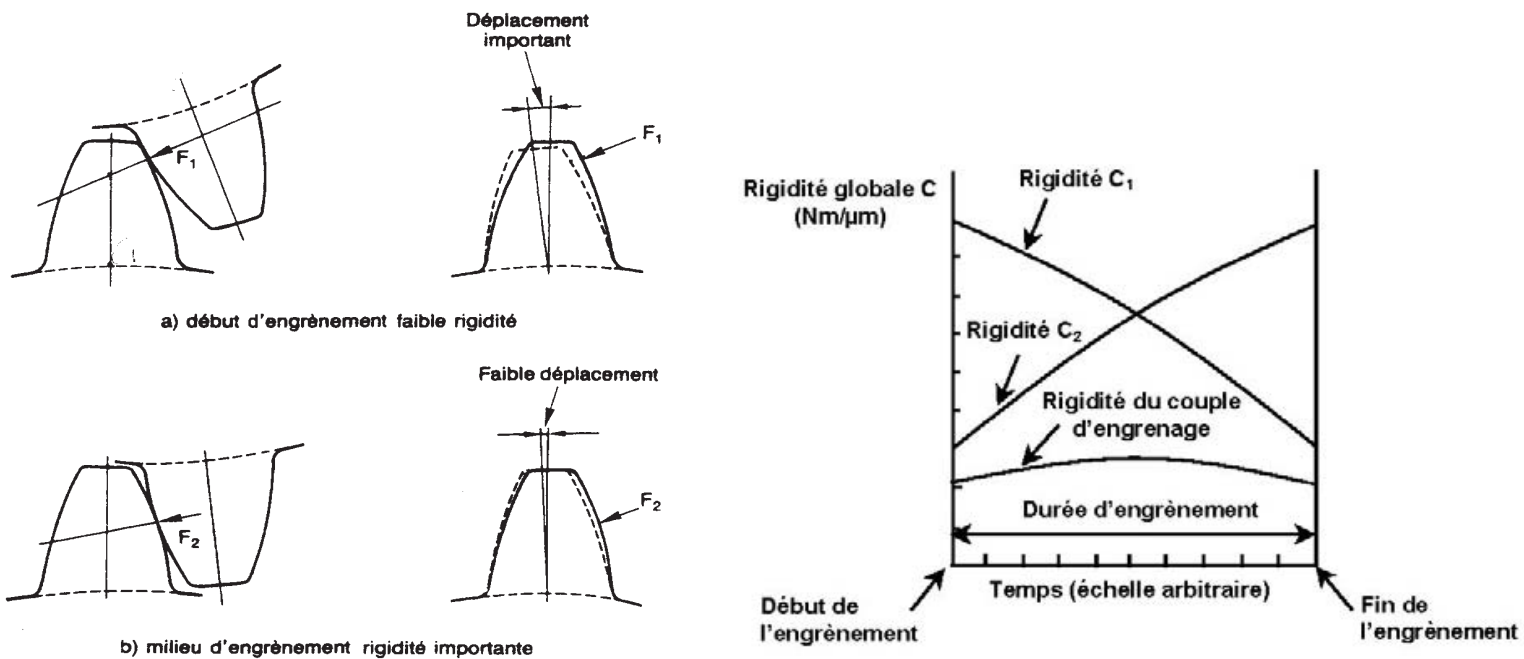

Fig. 1. Schéma de la cinématique de l'engrènement [1].

La deuxième décrit deux validations que nous avons réalisées en milieu industriel. L'une des applications porte sur un multiplicateur tournant à grande vitesse tandis que l'autre est effectuée sur un réducteur tournant à très faible vitesse.

\subsection{Analyse des mécanismes de génération des vibrations}

Dans un réducteur à engrenages, le mécanisme de génération des vibrations mécaniques met en jeu deux facteurs : les sources d'excitation et la transmission des vibrations aux parois du mécanisme.

Comme le montre la figure 1, les sources d'excitation proviennent principalement de l'impact entre les dents des deux roues lorsqu'elles engrènent et de la variation de la rigidité de la denture due au déplacement du point de tangence entre les profils des deux dents. Certaines excitations secondaires proviennent du changement de sens de la force de frottement au cours du contact de deux dents et de défauts géométriques liés à l'usinage des profils. On peut, le plus souvent, les relier à une caractéristique cinématique du réducteur. On retrouve ainsi, lors de l'analyse du spectre du signal capté sur le carter, les fréquences caractéristiques des éléments cinématiques.

Généralement, on distingue deux types de structures périodiques dans le spectre :

- les peignes de raies qui correspondent à des familles de composantes dont les fréquences $\mathrm{f}_{\mathrm{k}}$ sont des multiples entiers d'une fréquence de base $\mathrm{f}_{\mathrm{o}}: \mathrm{f}_{\mathrm{k}}=\mathrm{k} . \mathrm{f}_{\mathrm{o}}$;

- les familles de bandes latérales de modulation qui représentent les composantes de fréquences $\mathrm{f}_{\mathrm{po}}$ centrées de part et d'autre d'une composante de fréquence $\mathrm{f}_{\mathrm{o}}$ avec un pas d'espacement constant $\mathrm{f}_{\mathrm{m}}$ : $\mathrm{f}_{\mathrm{po}}=\mathrm{f}_{\mathrm{o}} \pm \mathrm{p} . \mathrm{f}_{\mathrm{m}},(\mathrm{p}$ : nombre entier positif $)$. Leur apparition est le plus souvent liée à des défauts de type « excentricité de denture » ou « jeu angulaire ».
En particulier, si le réducteur comporte un seul étage de réduction, on voit apparaître deux raies fondamentales correspondantes aux périodes de rotation $f_{1}$ et $f_{2}$ des arbres. La déformation du profil des dents lors de l'engrènement génère un signal périodique de fréquence $\mathrm{f}_{\mathrm{e}}=\mathrm{z}_{1} \cdot \mathrm{f}_{1}=\mathrm{z}_{2} \cdot \mathrm{f}_{2}$ avec $\mathrm{z}_{1}$ et $\mathrm{z}_{2}$ le nombre de dents du pignon et de la roue. Cette fréquence $f_{e}$ représente la source vibratoire principale appelée « signal d'engrènement ».

Un défaut de type «écaillage » ou «matage » produit également un choc au contact de la dent conjuguée et l'amplitude de la fréquence de la roue correspondante augmente. (Il apparaît également des bandes latérales qui résultent de la modulation de la fréquence d'engrènement par la fréquence de rotation de la roue portant le défaut.)

Le spectre typique renvoyé par un accéléromètre placé sur le carter d'un réducteur à engrenages devrait alors avoir l'allure de la figure 2a. Il n'en est pas souvent ainsi car ces vibrations sont transmises à travers le carter et excitent à leur tour les fréquences de résonance de celui-ci. S'ajoute à cela le bruit correspondant aux contributions indépendantes des caractéristiques cinématiques du mécanisme. La lecture de l'ensemble de ces interactions s'en trouve grandement compliquée et le signal obtenu en réalité a plutôt l'aspect de la figure $2 \mathrm{~b}$.

À partir de ce spectre, on peut effectuer une transformation du signal, appelée cepstre, dont on verra le détail dans le paragraphe suivant, et qui peut améliorer la situation :

- d'une part en séparant la partie du signal correspondant aux résonances de la structure excitée de la partie du signal correspondant aux éléments cinématiques;

- d'autre part en détectant dans le spectre un motif récurent correspondant à un choc cyclique.

La figure 2c montre le cepstre du signal de la figure $2 \mathrm{~b}$. On distingue nettement trois raies appelées rhamoniques (anagramme du mot harmoniques), appartenant à un peigne de raies décroissant, dont la première à $0.0054 \mathrm{~s}$ (soit $185 \mathrm{~Hz}$ ) correspond à la fréquence de rotation de 


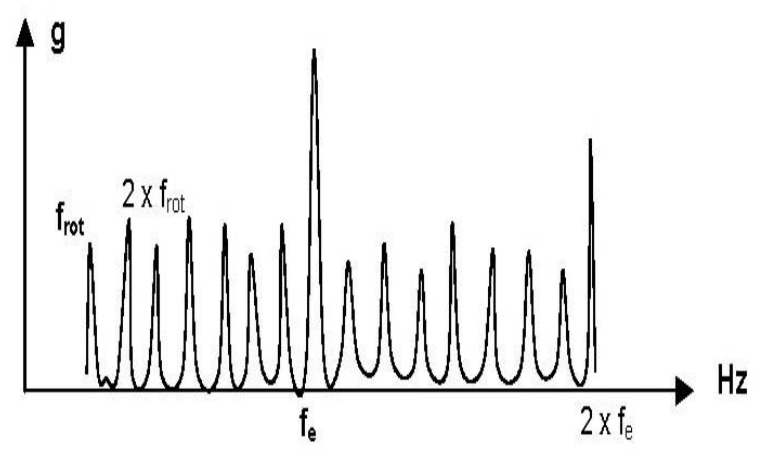

(a)

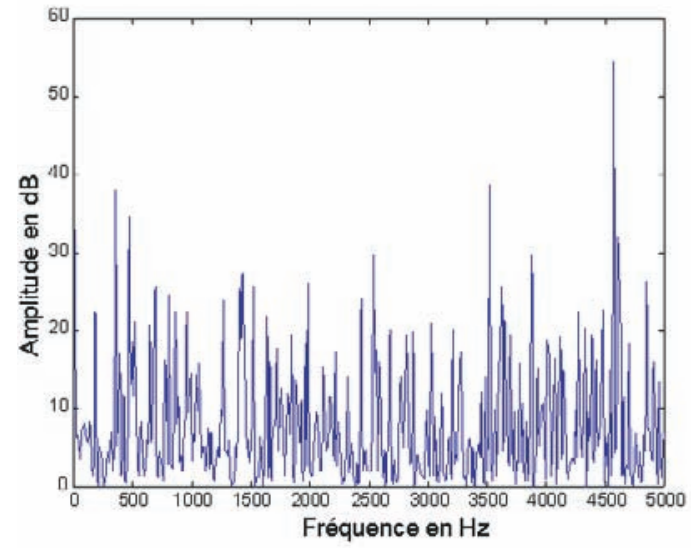

(b)

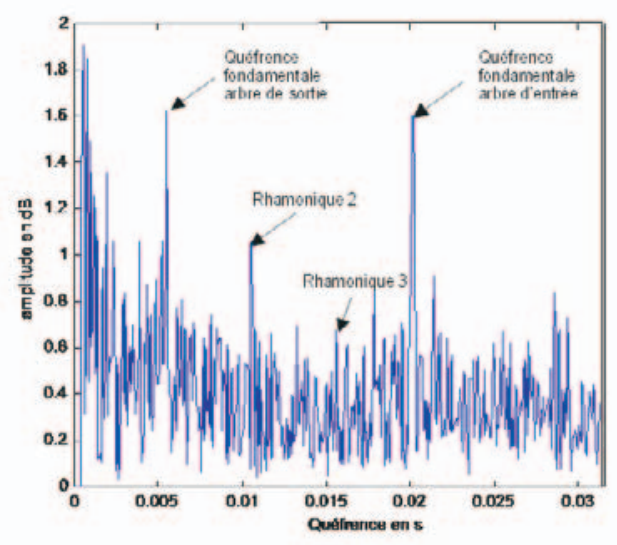

(c)

Fig. 2. (a) Schématisation du spectre correspondant à une dent détériorée. (b) Spectre de fréquence sur un multiplicateur industriel. (c) Cesptre sur multiplicateur industriel.

l'arbre de sortie du multiplicateur (caractéristique d'une dent écaillée sur le pignon de sortie). Le cepstre a donc la propriété d'identifier les structures périodiques spectrales, en particulier celles liées à des chocs périodiques.

Randall [2,3] montre que cet outil peut aussi être utilisé dans de nombreuses applications, en particulier en acoustique (signaux contenant des échos). Également Capdessus et Sidhamed [4] mettent en évidence sur un réducteur à engrenages dont les roues présentaient un nombre de dents très proche (20 et 21 dents), l'efficacité de cet outil en discernant deux raies de fréquences très proches, là où l'analyse spectrale n'en laissait apparaître qu'une. Enfin, El Badaoui et al. [5] ont montré que son efficacité pouvait être améliorée en effectuant une acquisition synchrone du signal.

\subsection{Aspects théoriques du cepstre}

Différentes approches théoriques ont été faites [4,6-8], et l'on peut retenir deux définitions de cet opérateur [6].

\section{Première définition (spectre complexe)}

Le cepstre d'un signal $s(t)$ peut être défini comme la transformée de Fourier inverse du logarithme décimal de sa transformée de Fourier directe :

$$
C[s(t)]=C(\tau)=T F^{-1}[\log T F[s(t)]]
$$

La variable $\tau$ du cepstre a la dimension d'un temps. Elle est appelée «quéfrence » (anagramme du mot fréquence). Elle représente les périodes d'oscillations des réponses impulsionnelles de la structure et les périodes de répétition ou de modulation des forces d'excitation.

\section{Deuxième définition (spectre de puissance)}

On définit également le cepstre comme la transformée de Fourier inverse du logarithme décimal du carré du module de sa transformée de Fourier :

$$
C[s(t)]=C(\tau)=T F^{-1} \log |T F[s(t)]|^{2}
$$

En comparant (2) avec la fonction d'autocorrélation :

$$
A c[s(t)]=A c(\tau)=T F^{-1}|T F[s(t)]|^{2}
$$


on voit que le logarithme du spectre est pris avant l'opération de transformée inverse. Il s'agit là d'un des principaux intérêts de cet outil pour notre application. En effet les signaux accélérométriques recueillis au niveau du capteur placé sur la structure surveillée caractérisent le signal vibratoire. Ils sont le produit de convolution d'une force d'excitation $e(t)$ par la réponse impulsionnelle $h(t)$ de la structure de la machine au point de fixation du capteur $[6,9]$ :

$$
S(t)=h(t) * e(t)
$$

Et d'après l'équation (1) on peut écrire :

$$
C[S(t)]=T F^{-1}[\log T F[h(t) * e(t)]]
$$

Le produit de convolution des transformées de Fourier de deux fonctions est égal au produit des transformées de Fourier de chacune d'elles, et donc l'équation (5) peut s'écrire :

$$
\begin{aligned}
C[S(t)]=T F^{-1}[\log [T F[ & h(t)]]] \\
& +T F^{-1}[\log [T F[e(t)]]]
\end{aligned}
$$

Le logarithme a pour effet de transformer la multiplication en addition et de séparer les signaux; il permet également de mettre en évidence les composantes spectrales de faibles amplitudes, contrairement à la fonction d'autocorrélation.

Le cepstre du signal vibratoire est donc la somme de deux cepstres :

$$
C[S(t)]=C[h(t)]+C[e(t)]
$$

- La fonction $C[h(t)]$ représentant le cepstre de la réponse impulsionnelle de la structure sur laquelle est fixée le capteur ;

- la fonction $C[e(t)]$ représentant le cepstre des forces d'excitation. C'est précisément cette partie qui nous intéresse.

En conséquence les amplitudes des composantes cepstrales liées aux forces d'excitation sont indépendantes de la position du capteur sur la structure; elles dépendent uniquement de la direction du mesurage. Cette propriété, anodine en laboratoire, prend toute son importance en milieu industriel où l'endroit le plus judicieux pour placer le capteur ne coïncide que rarement avec l'espace accessible de la machine.

Enfin, dans la grande majorité des cas, les périodes d'oscillations libres des résonances de structures excitées par des chocs sont généralement faibles devant les périodes de répétition des forces d'excitation.

Les informations issues de la réponse impulsionnelle se trouveront donc plutôt dans la partie gauche de la représentation cepstrale (haute fréquence), alors que celles propres aux forces d'excitation se situeront plutôt à partir de la zone centrale (voir Fig. 3).

L'amplitude d'une ou de plusieurs composantes cepstrales fondamentales est représentative de l'émergence d'une famille de composantes dans le spectre par rapport au bruit de fond. C'est l'analyse de chacune d'entre

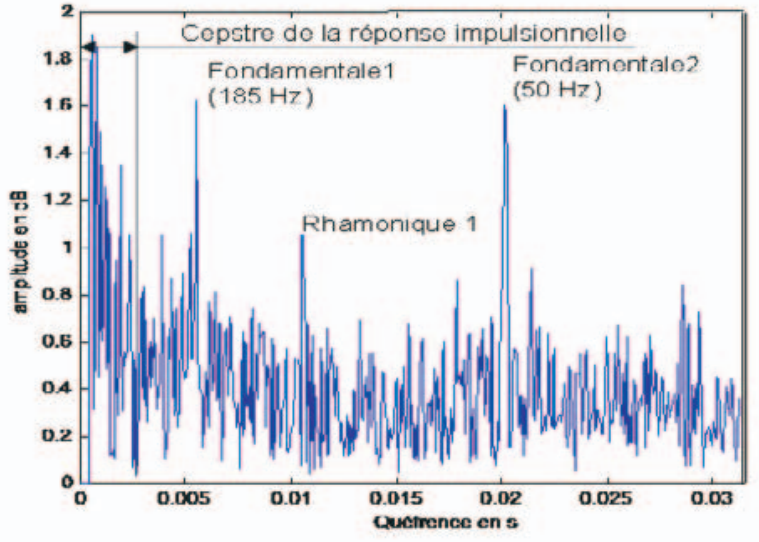

Fig. 3. Distinction sur un cepstre entre réponse impulsionnelle et forces d'excitation.

elles qui nous permet alors d'effectuer un diagnostic. À l'intérieur d'une même famille, on observe une décroissance rapide de l'amplitude des composantes ou rhamoniques, en $1 / \mathrm{n}$ ( $\mathrm{n}$ étant l'ordre de la rhamonique, voir Fig. 4). Ceci est la conséquence de la déformation du signal par la fenêtre d'apodisation utilisée pour calculer le spectre de base [9]. On ne peut pas attribuer une signification physique à l'amplitude des rhamoniques, mais leur espacement est constant et correspond à une caractéristique cinématique du mécanisme surveillé.

\subsection{Proposition d'un méthodologie de paramétrage du cepstre}

Le paramétrage d'une mesure cepstrale sur un analyseur doit être réalisé à partir du choix d'une plage de fréquences. Ce choix est primordial car il conditionne le fait de visualiser, ou non, le défaut que l'on cherche à observer. Il n'existe pas de règle pour aider au choix de cette plage de fréquence. Nous avons donc créé des tables (en fonction de la résolution du collecteur) qui permettent, en situation industrielle, de paramétrer rapidement et de manière sure une mesure cepstrale.

Le but recherché est de définir, à partir de la connaissance de la fréquence du phénomène périodique que l'on veut observer, la plage de fréquence maximum $\mathrm{f}_{\operatorname{maxi}}$ pour l'acquisition de notre cepstre qui va permettre d'obtenir une résolution optimisée tout en conservant les deux premières rhamoniques.

Résolution du collecteur : $\mathrm{R}_{\mathrm{e}}$ (400, 800, 1600, 3200 lignes).

Fréquence d'échantillonnage $\mathrm{du}$ collecteur $\mathrm{f}_{\mathrm{e}}=$ 2,56 $\mathrm{f}_{\text {maxi }}$ (théorème de Shannon).

La fréquence à partir de laquelle on commence la mesure ou fréquence de départ est $f_{d}=f_{e} / R_{e}$.

On en déduit la quéfrence de départ $q_{d}=1 / f_{d}$, (c'est la quéfrence maximale que l'on enregistrera). La quéfrence suivante est obtenue en retranchant à la quéfrence précédente le terme $1 / \mathrm{f}_{\mathrm{e}}$. 


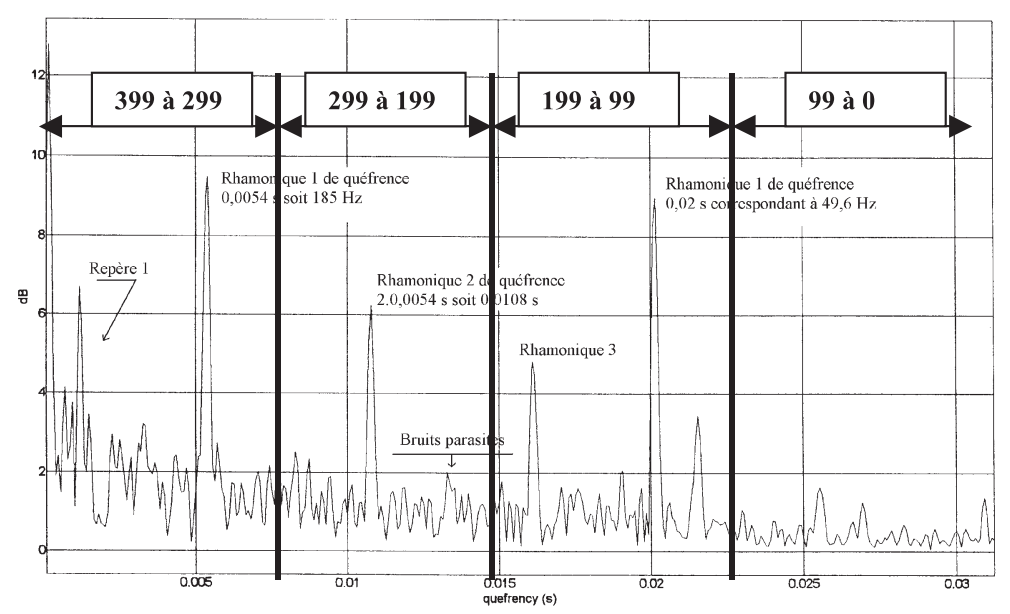

Fig. 4. Cepstre paramétré sur $5000 \mathrm{~Hz}$.

\begin{tabular}{|c|c|c|c|c|c|c|}
\hline \multirow{2}{*}{$\begin{array}{l}\text { Fréquence } \\
\text { Maximum } \\
\qquad \mathrm{Hz}\end{array}$} & \multirow{2}{*}{$\begin{array}{c}\text { Fréquence } \\
\text { d'échantillonnage } \\
\mathrm{Hz}\end{array}$} & \multirow{2}{*}{$\begin{array}{c}\text { Fréquence de } \\
\text { départ } \\
\mathrm{Hz}\end{array}$} & \multirow{2}{*}{$\begin{array}{c}\text { Fréquence } \\
\text { finale } \\
\mathbf{H z}\end{array}$} & \multicolumn{3}{|c|}{ Rhamoniques } \\
\hline & & & & $\begin{array}{c}\mathrm{n}^{\circ} 1 \\
(\mathrm{n}=299) \\
\mathrm{Hz}\end{array}$ & $\begin{array}{c}\mathbf{n}^{\circ} 2 \\
(\mathbf{n}=199) \\
\mathbf{H z}\end{array}$ & $\begin{array}{c}\mathbf{n}^{\circ} 3 \\
(\mathbf{n}=99) \\
\mathrm{Hz} \\
\end{array}$ \\
\hline 10 & 25.6 & 0.064 & 25.6 & 0.3 & 0.1 & 0.1 \\
\hline 20 & 51.2 & 0.128 & 51.2 & 0.5 & 0.3 & 0.2 \\
\hline 50 & 128 & 0.32 & 128 & 1.3 & 0.6 & 0.4 \\
\hline 100 & 256 & 0.64 & 256 & 2.5 & 1.3 & 0.9 \\
\hline 200 & 512 & 1.28 & 512 & 5.1 & 2.5 & 1.7 \\
\hline 500 & 1280 & 3.2 & 1280 & 12.7 & 6.4 & 4.3 \\
\hline 1000 & 2560 & 6.4 & 2560 & 25.3 & 12.7 & 8.5 \\
\hline 2000 & 5120 & 12.8 & 5120 & 50.7 & 25.5 & 17 \\
\hline 5000 & 12800 & 32 & 12800 & 126.7 & 63.7 & 42.5 \\
\hline 10000 & 25600 & 64 & 25600 & 253.5 & 128.0 & 85 \\
\hline 20000 & 51200 & 128 & 51200 & 506.9 & 254.7 & 170.1 \\
\hline 40000 & 102400 & 256 & 102400 & 1013.9 & 509.5 & 340.2 \\
\hline
\end{tabular}

Fig. 5. Table de paramétrage du cepstre pour une résolution de 400 lignes.

On obtient alors une suite arithmétique de premier terme $\mathrm{q}_{\mathrm{d}}$, et de raison $\mathrm{r}=-1 / \mathrm{f}_{\mathrm{e}}$.

Ainsi pour $R_{e}=400$ lignes.

q399 est le $400^{\mathrm{e}}$ et dernier terme de cette suite et a pour valeur : 1/fe (c'est la quéfrence minimale que l'on enregistrera). Un terme quelconque est $\mathrm{q}_{\mathrm{n}}=\mathrm{q}_{\mathrm{d}}+$ n.r.

Par exemple, le cepstre de la figure 4 ci-dessous est paramétré avec une fréquence $\mathrm{f}_{\operatorname{maxi}}=5000 \mathrm{~Hz}$; soit une fréquence d'échantillonnage $\mathrm{f}_{\mathrm{e}}=12800 \mathrm{~Hz}$; la raison est $\mathrm{r}=-1 / 12800 \mathrm{~Hz}$ et la fréquence de départ $\mathrm{f}_{\mathrm{d}}=32 \mathrm{~Hz}$ pour $\mathrm{R}_{\mathrm{e}}=400$ lignes.

La fréquence $\mathrm{f}=185 \mathrm{~Hz}$ donne $\mathrm{q}_{\mathrm{n}}=1 / 185=$ $0,005405 \mathrm{~s}$.

Le terme correspondant est le $331^{\mathrm{e}}$ de la suite arithmétique $\mathrm{q}_{\mathrm{n}}=1 / 32+\mathrm{n}(-1 / 12800)$.

Afin d'être en mesure de visualiser la quéfrence fondamentale ainsi que deux de ses rhamoniques nous divisons notre représentation cepstrale en quatre zones :

$-1^{\text {re }}$ zone jusqu'à $\mathrm{n}=99$; elle doit contenir la quéfrence correspondant à la rhamonique 3 ; $-2^{\mathrm{e}}$ zone de $\mathrm{n}=99$ jusqu'à $\mathrm{n}=199$; elle doit contenir la quéfrence correspondant à la rhamonique 2 ;

$-3^{\text {e }}$ zone de $\mathrm{n}=199$ jusqu'à $\mathrm{n}=299$; elle doit contenir la quéfrence correspondant à la rhamonique 1 ou fondamentale;

$-4^{\mathrm{e}}$ zone $:$ de $\mathrm{n}=299$ jusqu'à $\mathrm{n}=399$; elle contient en général la réponse impulsionnelle de la structure.

On définit alors des tables de paramétrage pour différentes résolutions (400, 800, 1600, 3200 lignes) comme dans la figure 5.

Pour le paramétrage du collecteur, il faut chercher dans la figure 5 la fréquence la plus proche de la fréquence fondamentale caractérisant le défaut dans la colonne Rhamonique $\mathrm{n}^{\circ} 1$, et lui associer « la gamme de fréquence maximum $»$ correspondante.

Par exemple, pour un réducteur avec $\omega_{\text {entrée }}=$ 3000 tr. $\min ^{-1}$ et $\omega_{\text {sortie }}=11220$ tr. $\mathrm{min}^{-1}$.

Un écaillage sur une dent de l'arbre d'entrée va générer un choc une fois par tour à la fréquence de $50 \mathrm{~Hz}$, 
la gamme de fréquence du cepstre sera donc de $2000 \mathrm{~Hz}$ (Fig. 5).

Un écaillage sur une dent de l'arbre de sortie va générer un choc une fois par tour à la fréquence de $187 \mathrm{~Hz}$, la gamme de fréquence du cepstre sera donc de $5000 \mathrm{~Hz}$ (on choisit la fréquence la plus proche).

\section{Validation de l'analyse cepstrale en milieu industriel}

Nous présentons deux applications de l'analyse cepstrale que nous avons réalisé en milieu industriel.

La première concerne un diagnostic par comparaison de 4 multiplicateurs de vitesse de forte puissance dans une usine agroalimentaire. On teste l'efficacité du cepstre pour la détection de défauts sur des systèmes à engrenages tournant à grande vitesse (application $\mathrm{n}^{\circ} 1$ ).

La deuxième présente une expertise réalisée sur un réducteur entraînant un broyeur dans une usine de fabrication d'engrais agricole. On teste l'efficacité du cepstre pour la détection de défauts sur des systèmes à engrenages tournant à très faible vitesse (application $\mathrm{n}^{\circ} 2$ ).

Pour ces deux applications le matériel de mesure était :

- un collecteur Brüel\&Kjaer type 2526 avec le logiciel d'analyse Sentinel ;

- un collecteur Stell Diagnostic type Movilog2 avec le logiciel DivaDiag.

\subsection{Diagnostic par analyse cepstrale d'un multiplicateur de vitesse}

\subsubsection{Descriptif de l'installation et contexte de mesure}

Les multiplicateurs entraînent des compresseurs centrifuges à roues (Fig. 6), fonctionnant en continu 350 jours par an, destinés à faire de la re-compression de vapeur. Chacun d'eux est composé d'un seul étage d'engrenages à denture hélicoïdale guidé par des paliers fluides. Ils sont entraînés par des moteurs de $860 \mathrm{Kw}$ à 3000 tr.min ${ }^{-1}$ $(50 \mathrm{~Hz})$.

Sept séries de mesures ont été effectuées sur une période de six mois sur les multiplicateurs. Les mesures cepstrales ont toujours fait apparaître le défaut mais nous n'avons pas constaté d'évolution de celui-ci au cours des différentes séries de mesures. C'est la comparaison des cepstres sur les 4 multiplicateurs qui nous a permis de construire notre diagnostic sur le multiplicateur $n^{\circ} 2$.

\subsection{2 Étude cinématique de l'installation (multiplicateur)}

- Vitesse de rotation de l'arbre d'entrée du multiplicateur : 3000 tr.min ${ }^{-1}(50 \mathrm{~Hz})$

- Vitesse de rotation de l'arbre de sortie : 11214 tr. $\mathrm{min}^{-1}(187 \mathrm{~Hz})$

- Nombre de dents du pignon menant : 157 dents

- Nombre de dents du pignon mené : 42 dents

- Fréquence d'engrènement : $7850 \mathrm{~Hz}$.
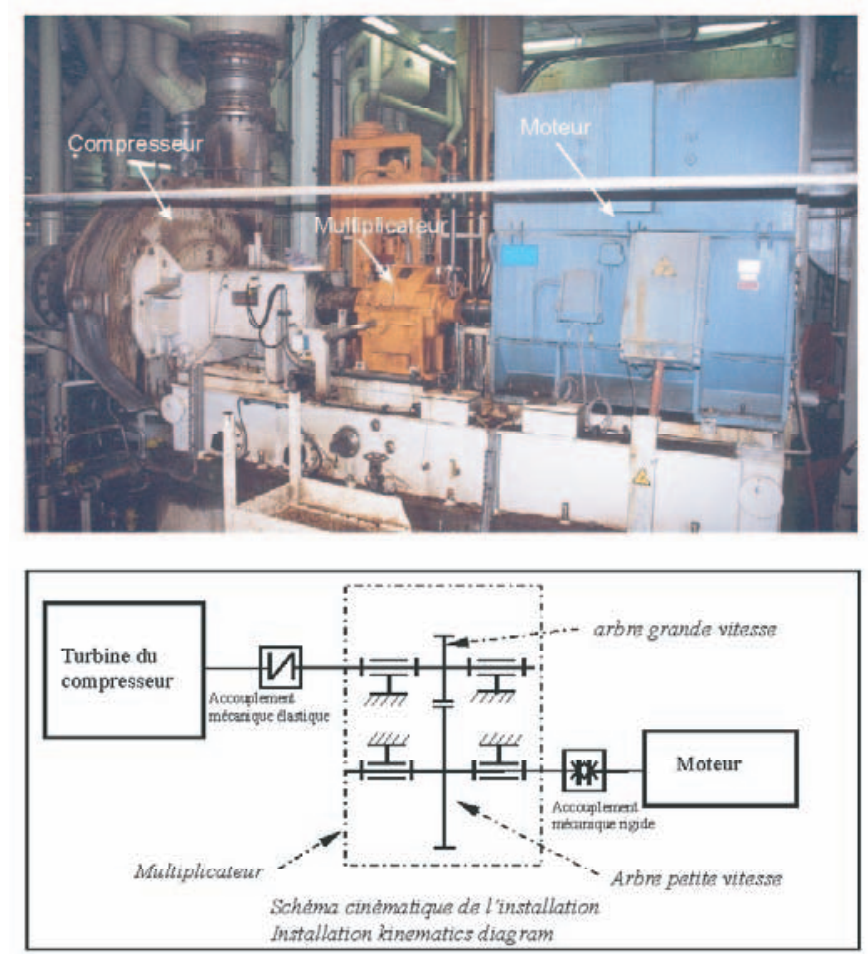

Fig. 6. Photo d'un compresseur « RMV».

\subsubsection{Paramétrage du collecteur et choix des points} de mesures

Nous avons fait le choix de toujours travailler sur 400 lignes (1024 points) afin de garder des temps d'acquisition « raisonnables » pour de la mesure industrielle.

Le paramètre mesuré a été la valeur RMS de l'accélération.

\begin{tabular}{cccc}
\hline $\begin{array}{c}\text { Plage } \\
\text { de } \\
\text { fréquences }\end{array}$ & $\begin{array}{c}\text { Nombre } \\
\text { de } \\
\text { points }\end{array}$ & $\begin{array}{c}\text { Temps de mesure } \\
\text { théorique pour }\end{array}$ & $\begin{array}{c}\text { Temps de mesure } \\
\text { pratique pour }\end{array}$ \\
\hline \multirow{2}{*}{$2000 \mathrm{~Hz}$} & 1024 & $\begin{array}{c}0.2 \text { seconde } \\
\text { 10 moyennes }\end{array}$ & $\begin{array}{c}6.7 \text { secondes } \\
\end{array}$ \\
& 2048 & 0.4 seconde & 13.4 secondes \\
\hline \multirow{2}{*}{$5000 \mathrm{~Hz}$} & 1024 & 0.08 seconde & 5.7 secondes \\
& 2048 & 0.16 seconde & 11.4 secondes \\
\hline
\end{tabular}

Le train d'engrenages du multiplicateur peut présenter les défauts suivants :

- un ou plusieurs écaillages sur le pignon de l'arbre d'entrée et de sortie;

- une usure généralisée de la denture;

- un engrènement en fond de denture.

Par conséquent les fréquences à suivre sont (Fig. 6) :

- la fréquence de rotation de l'arbre d'entrée $(50 \mathrm{~Hz})$ et ses harmoniques;

- la fréquence de l'arbre de sortie $(187 \mathrm{~Hz})$ et ses harmoniques;

- la fréquence d'engrènement $(7850 \mathrm{~Hz})$ et ses harmoniques. 


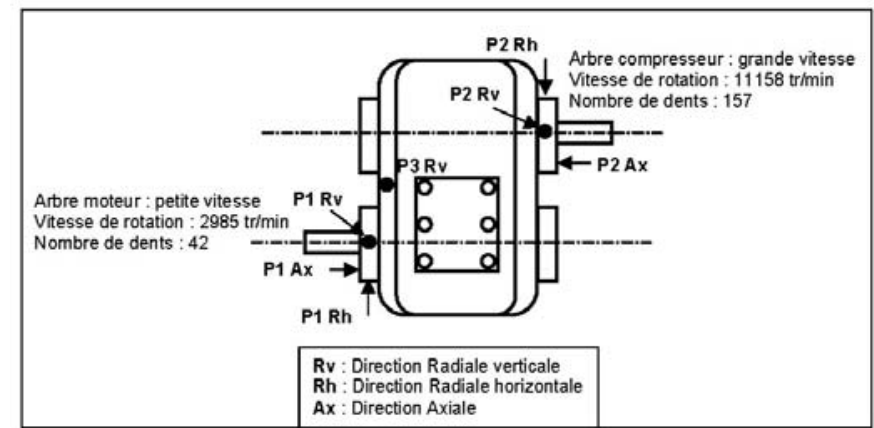

Fig. 7. Position des points de mesures vibratoires sur le multiplicateur.

Nous avons réalisé des mesures globales en vitesse efficace $(10-1000 \mathrm{~Hz})$, en accélération efficace $(10-10000 \mathrm{~Hz})$ ainsi que des mesures fréquentielles sur 0-200 Hz, 0-1000 Hz, 0-10 kHz et 0-20 kHz.

L'utilisation de la table de paramétrage nous a conduit à paramétrer des cepstres sur 0-2000 Hz (pour la surveillance de la fréquence à $50 \mathrm{~Hz}$ ) et $0-5000 \mathrm{~Hz}$ (pour la surveillance de la fréquence à $185 \mathrm{~Hz}$ ).

Le suivi de la fréquence d'engrènement par l'analyse cepstrale n'a pas été possible car la gamme de fréquences à sélectionner dépassait la capacité du collecteur $(>40 \mathrm{kHz})$.

Nous avons disposé l'accéléromètre suivant deux directions (radiale horizontale et verticale) en trois positions différentes sur le multiplicateur comme indiqué sur la figure 7 .

\section{Influence de la position du capteur sur la structure}

Les cepstres se superposent et cette observation s'est vérifiée pour les sept campagnes de mesures.

Les trois cepstres ci-dessous montrent que l'on retrouve bien la même signature vibratoire quelle que soit la position du point de mesure.

L'amplitude de la quéfrence fondamentale du dernier cepstre sur le point P3 est très largement supérieure aux deux autres : $22 \mathrm{~dB}$ pour $12 \mathrm{~dB}$ (Figs. 8-10).

C'est sur ce point P3 que nous avons décidé de réaliser le suivi des multiplicateurs.

Les raisons de ce choix sont les suivantes:

- c'est en ce point que l'on observe le rapport signal sur bruit le plus favorable:

- c'est le point le plus facilement accessible sur cette installation (éloignement des parties tournantes).

\subsubsection{Comparaison des résultats d'une analyse fréquentielle et d'une analyse cepstrale}

Les enregistrements qui suivent portent sur le traitement des mesures de la sixième campagne.

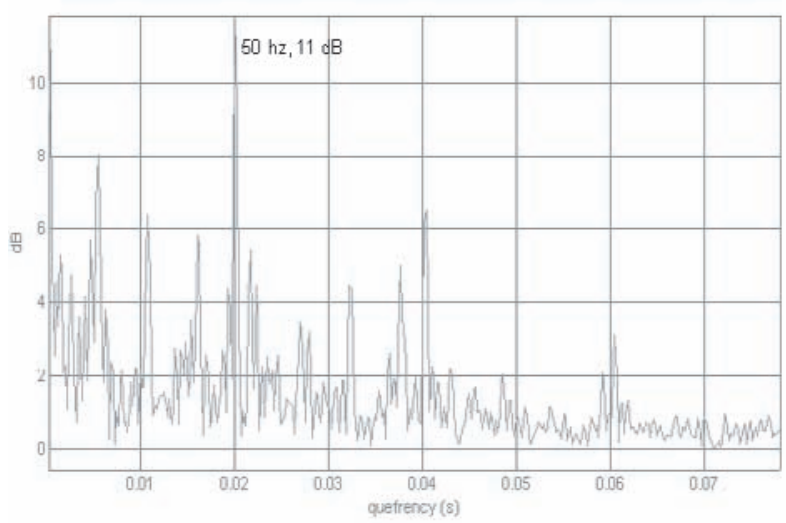

Fig. 8. Multiplicateur $\mathrm{n}^{\circ} 2-$ Point P1 position radiale horizontale.

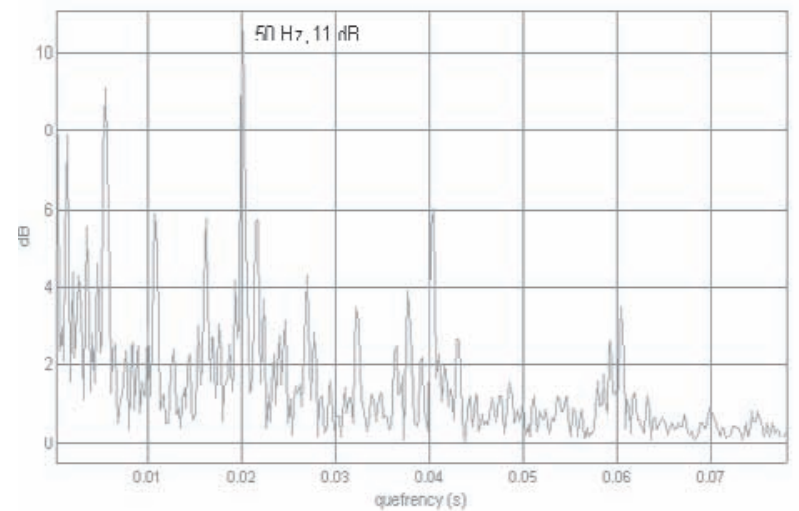

Fig. 9. Multiplicateur $\mathrm{n}^{\circ} 2-$ Point $\mathrm{P} 2$ position radiale horizontale.

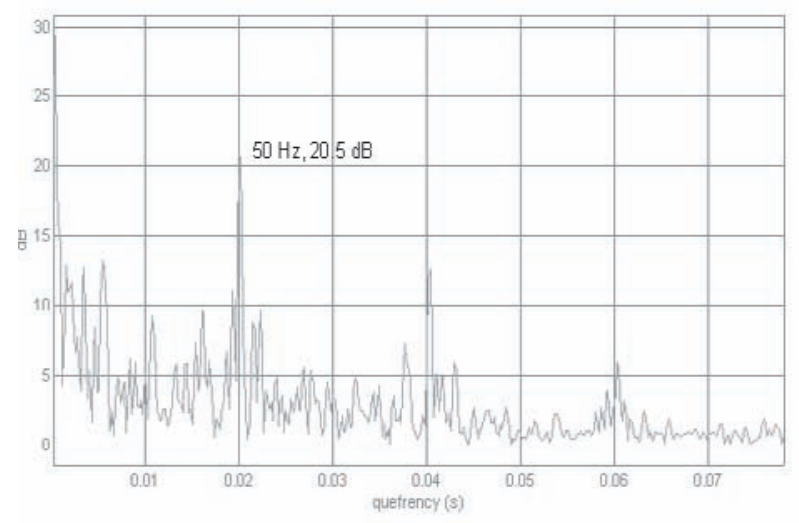

Fig. 10. Multiplicateur $\mathrm{n}^{\circ} 2-$ Point P3 position radiale horizontale.

\subsubsection{Analyse des spectres}

Le spectre de la figure 11 fait apparaître la fréquence de l'arbre menant du multiplicateur (50 Hz avec une amplitude à $0,0052 \mathrm{~g}$ ) et la fréquence de l'arbre mené $(186,11 \mathrm{~Hz}$ avec une amplitude à $0,072 \mathrm{~g})$. Leur amplitude ne laisse présager d'aucun phénomène particulier. 


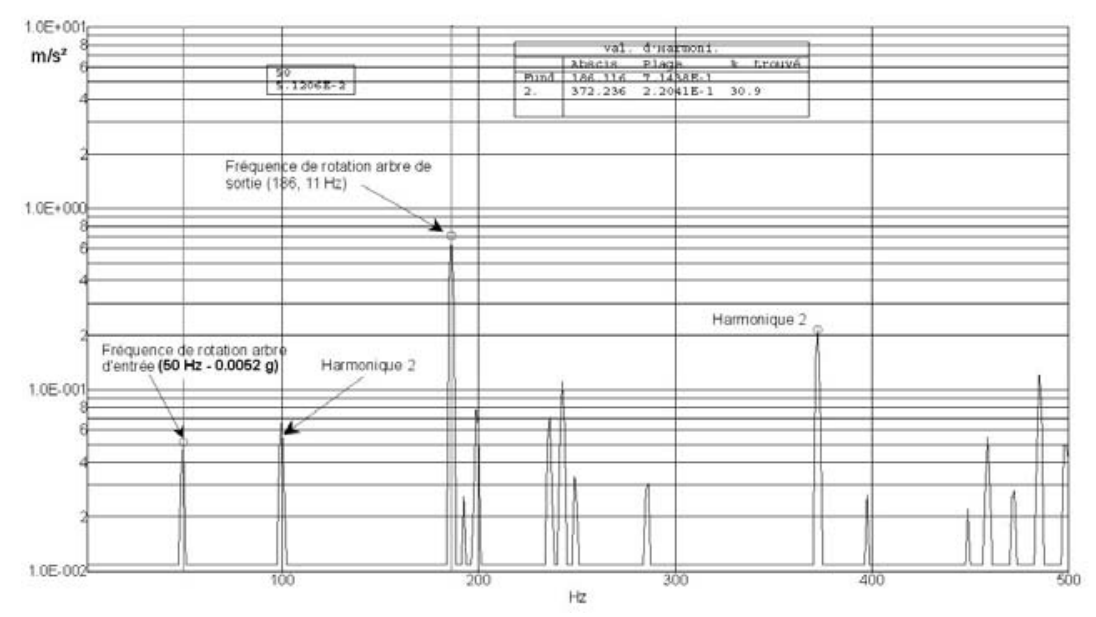

Fig. 11. Multiplicateur $n^{\circ} 1$ - Spectre sur $500 \mathrm{~Hz}$ (échelle log).

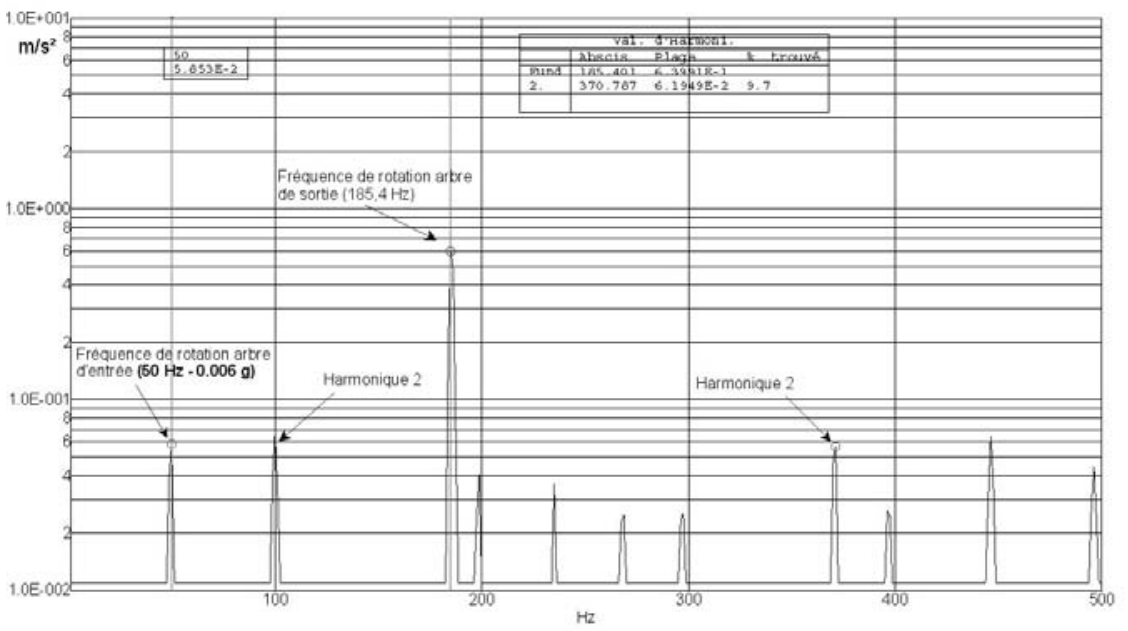

Fig. 12. Multiplicateur $\mathrm{n}^{\circ} 2$ - Spectre sur $500 \mathrm{~Hz}$ (échelle log).

À présent, si l'on compare les spectres sur $0-500 \mathrm{~Hz}$ des multiplicateurs $n^{\circ} 1$ et $n^{\circ} 2$ (Figs. 11 et 12), on voit que l'amplitude de la fréquence de rotation de l'arbre menant $(50 \mathrm{~Hz})$ est peut différente d'un multiplicateur à l'autre $(0,0052$ et $0,006 \mathrm{~g})$.

Sans l'utilisation d'un zoom puissant autour de la fréquence d'engrènement il est impossible de discerner sur le spectre de la figure 13 la présence de familles de bandes latérales.

\subsubsection{Analyse des cepstres}

Le cepstre sur $2000 \mathrm{~Hz}$ du multiplicateur n ${ }^{\circ} 1$ (Fig. 14) présente un faible peigne de raies associé aux quéfrences de $0.0053 \mathrm{~s}(187 \mathrm{~Hz})$ et $0.02 \mathrm{~s}(50 \mathrm{~Hz})$.

Le cepstre de la figure 15 fait clairement apparaitre sur le multiplicateur $n^{\circ} 2$ un peigne de raies significatif associé à la quéfrence de $0.02 \mathrm{~s}$ (amplitude multipliée par quatre par rapport au multiplicateur $\mathrm{n}^{\circ} 1$ ). On obtient la séparation de la partie intéressante du signal de la partie correspondante à la réponse de la structure ainsi que la décroissance en $1 / \mathrm{n}$ décrite dans la première partie de ce travail.

La comparaison des cepstres des 4 multiplicateurs nous a permis de construire notre diagnostic sur le multiplicateur $n^{\circ} 2$.

On peut diagnostiquer un choc à la fréquence de $50 \mathrm{~Hz}$ caractéristique d'un défaut de type «écaillage » sur une dent du pignon menant du multiplicateur. Nous n'avons pas constaté de phénomène de rodage du défaut (le choc n'a pas présenté d'atténuation au cours des différentes campagnes de mesure).

L'intérêt de l'utilisation de l'analyse cepstrale est ici évident, car en une seule campagne de mesure nous avons pu identifier un risque potentiel de défaut. Le recours de manière systématique au zoom aurait considérablement augmenté les temps d'acquisition, (multiplié par 5) et l'emploi des techniques de démodulation (spectre d'enveloppe) nécessitait systématiquement une deuxième campagne de mesure (identification des phénomènes de résonance). 


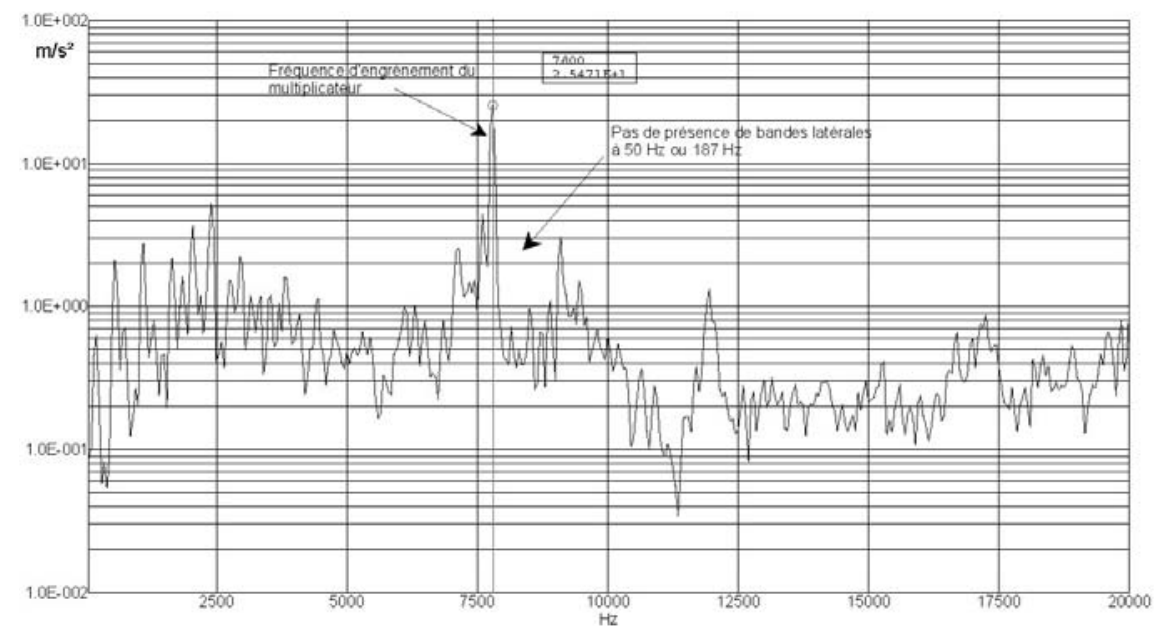

Fig. 13. Multiplicateur $n^{\circ} 2$ - Spectre sur $20000 \mathrm{~Hz}$ (échelle log).

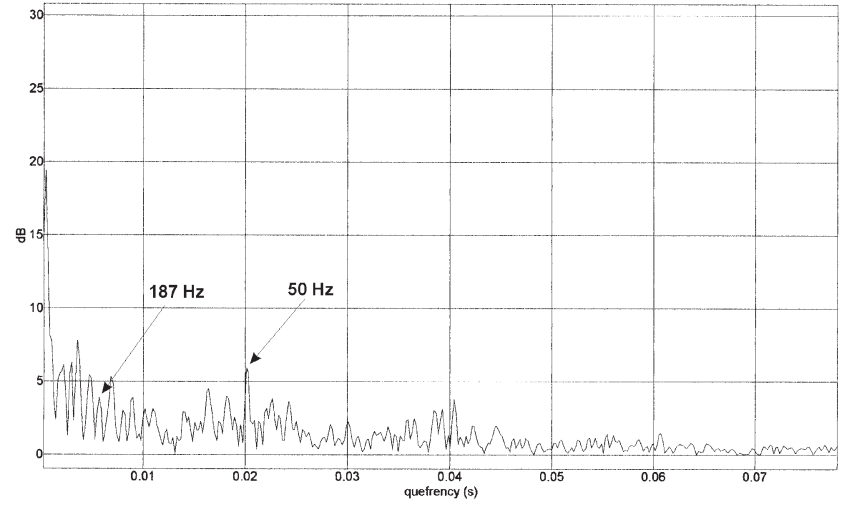

Fig. 14. Multiplicateur $n^{\circ} 1-$ Cepstre sur $2000 \mathrm{~Hz}$.

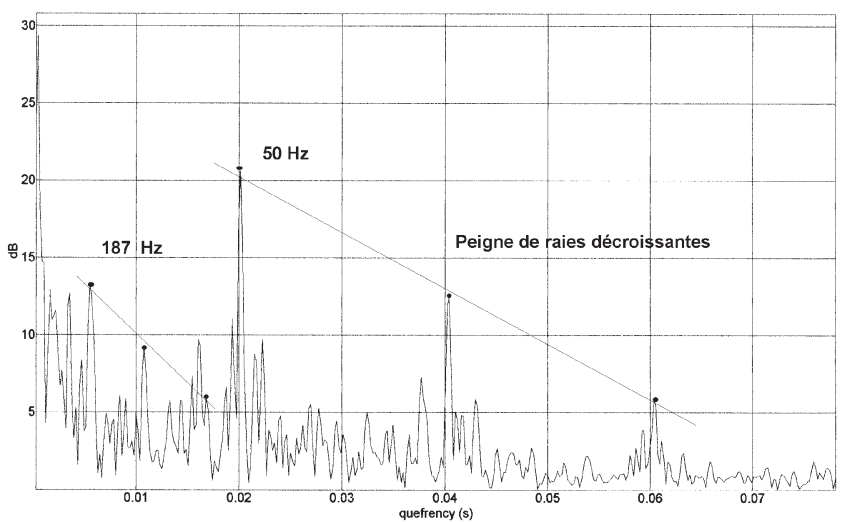

Fig. 15. Multiplicateur $n^{\circ} 2$ - Cepstre sur $2000 \mathrm{~Hz}$.

\subsubsection{Confirmation du diagnostic}

Le réducteur a été arrêté et ouvert à la suite de ce diagnostic afin de vérifier l'état du pignon. Cette intervention a permis de confirmer l'écaillage de la dent du pignon menant et de prévoir une opération de maintenance.

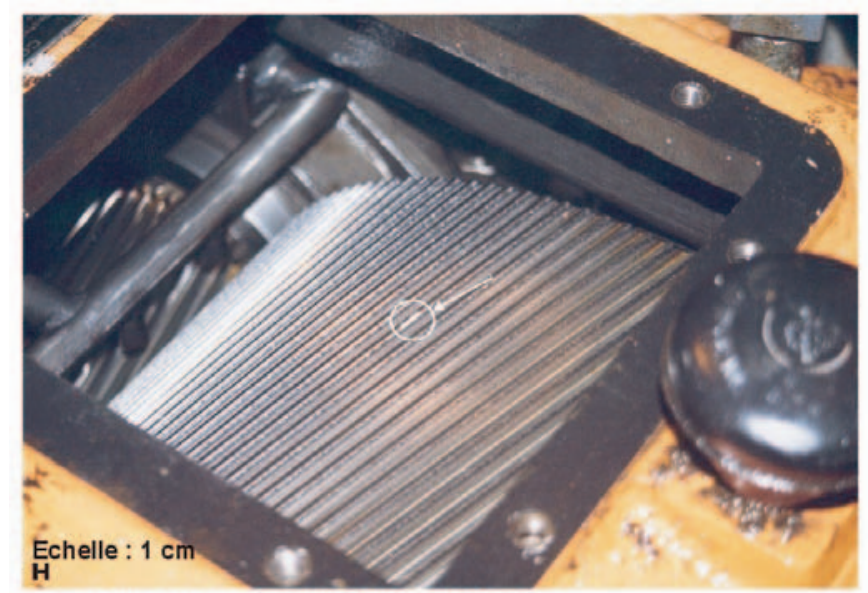

Fig. 16. Photographie de la dent détériorée par écaillage.

\subsection{Diagnostic par analyse cepstrale d'un réducteur entraînant un broyeur}

\subsubsection{Descriptif de l'installation et contexte de mesure}

Cette deuxième application présente un suivi vibratoire réalisé sur un réducteur de forte puissance (300 kw) à double sorties, composé de trois trains d'engrenages à denture hélicoïdale (Fig. 17). Il entraîne un broyeur à meules dans une usine de fabrication d'engrais agricole et fonctionne en continu 350 jours par an.

Ce cas est intéressant car il regroupe les conditions suivantes :

- la cinématique du réducteur est complexe;

- la vitesse de rotation des arbres de sortie du réducteur est faible $\left(30\right.$ tr. $\left.\mathrm{min}^{-1}\right)$;

- le milieu est bruité (l'ensemble repose sur un châssis métallique).

Nous avons eu «la chance » de pouvoir assister à l'évolution d'une dégradation sur la denture de ce réducteur. 


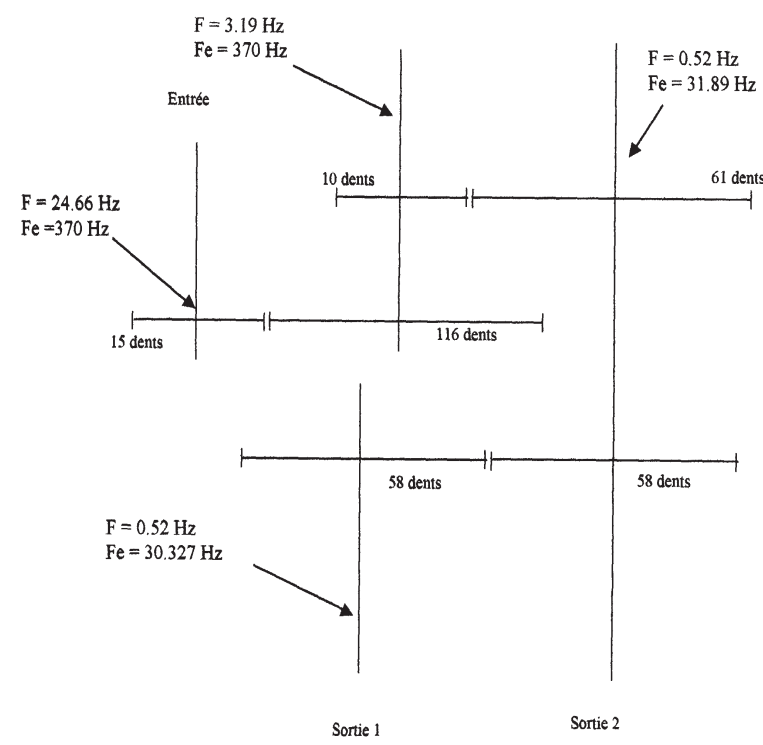

Fig. 17. Identification des fréquences caractéristiques des défauts sur les engrenages du réducteur.

\subsection{2 Étude cinématique de l'installation}

- Vitesse de rotation de l'arbre d'entrée du multiplicateur : 1480 tr. min $^{-1}(24,6 \mathrm{~Hz})$.

- Vitesse de rotation de l'arbre de sortie : $31,37 \mathrm{tr} \cdot \mathrm{min}^{-1}$ $(0,52 \mathrm{~Hz})$.

- Identification des fréquences de coïncidence des engrenages :

\begin{tabular}{ll}
\hline Couple de pignons 15 et 116 dents & $0,42 \mathrm{~Hz}$ \\
Couple de pignons 10 et 61 dents & $0,6 \mathrm{~Hz}$ \\
Couple de pignons 58 et 58 dents & $0,52 \mathrm{~Hz}$ \\
\hline
\end{tabular}

\subsubsection{Paramétrage du collecteur et choix des points de mesures}

Les cepstres ont été paramétrés avec la table de paramétrage proposée dans la première partie, sur 400 lignes (Fig. 5).

Les mesures fréquentielles ont été paramétrées avec l'objectif de visualiser les fréquences de rotation et d'engrènement des différents trains d'engrenages.

Comme dans l'étude précédente, la sensibilité du point d'implantation du capteur a été analysée. L'accéléromètre a été placé au plus près des parties tournantes (en général sur les paliers) suivant les directions radiale horizontale, verticale et axiale indiquées sur la figure 18.

\subsubsection{Comparaison des résultats d'une analyse fréquentielle et d'une analyse cepstrale}

\subsubsection{Mesures réalisées sur le premier train d'engrenages}

\subsection{Analyse des spectres}

Les spectres des figures 19 et 20 correspondent aux mesures réalisées avant et pendant la détérioration des

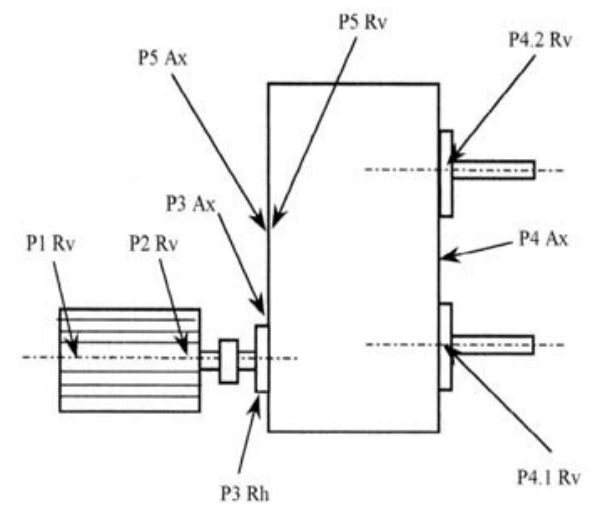

Fig. 18. Position et direction des points de mesure.

trains d'engrenages du réducteur sur le point P3 (voir Fig. 18) en position radiale horizontale.

L'amplitude de la fréquence de rotation de l'arbre d'entrée du réducteur a évolué de $0,002 \mathrm{~g}$ à $0,059 \mathrm{~g}$ et celle de la fréquence d'engrènement de $0,01 \mathrm{~g}$ à $0,025 \mathrm{~g}$.

Le spectre de la figure 20 fait apparaitre des bandes latérales centrées autour de la fréquence d'engrènement au pas de $24,6 \mathrm{~Hz}$.

La comparaison de ces deux spectres amène à penser à un risque d'arrachement ou d'écaillage d'une dent sur le pignon 15 dents d'entrée du réducteur (choc une fois par tour du pignon).

\subsection{Analyse des cepstres}

Le diagnostic est totalement validé par les cepstres des figures 21 et 22 .

On constate que la comparaison des cepstres est beaucoup plus aisée et qu'ils se prêtent bien à l'utilisation de gabarits.

Dans cet exemple, l'analyse cepstrale permet d'apporter une validation du diagnostic obtenu à l'aide des mesures fréquentielles. Elle se justifie en regard de l'importance de la machine dans le processus de production.

L'évolution de l'amplitude des quéfrences fondamentales a été très rapide. Les enregistrements intermédiaires ont présenté une augmentation régulière de la quéfrence. Leur faible nombre (trois) ne permettent pas de fournir une courbe de tendance assez précise.

\subsubsection{Mesures réalisées sur le deuxième et le troisième train d'engrenages}

\subsection{Analyse des spectres}

Le diagnostic par mesures fréquentielles est ici beaucoup plus délicat car les phénomènes mécaniques à observer sont en très basses fréquences (inférieur à $3,5 \mathrm{~Hz}$ ).

Le spectre de la figure 23 montre qu'en dessous de $10 \mathrm{~Hz}$ l'accéléromètre n'est plus assez sensible pour enregistrer un signal vibratoire significatif. Il est impossible de distinguer sur ce spectre la fréquence de rotation de l'arbre intermédiaire $(3,2 \mathrm{~Hz})$ et de l'arbre 


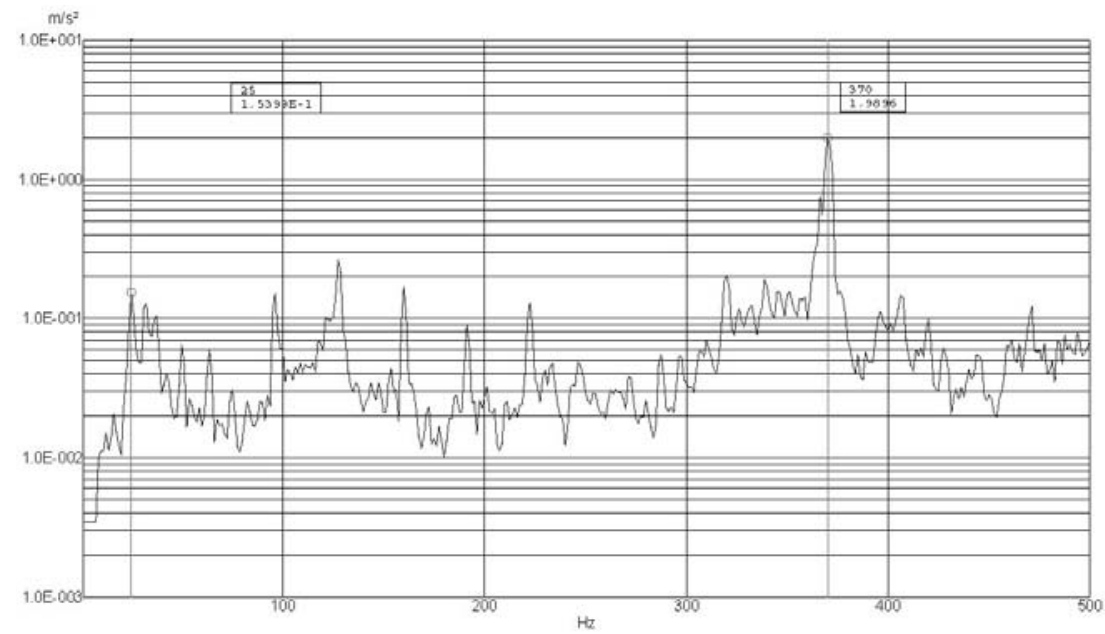

Fig. 19. Point de mesure $\mathrm{P} 3 \mathrm{Rh}-$ Spectre $0-500 \mathrm{~Hz}$ réducteur sain (échelle log).

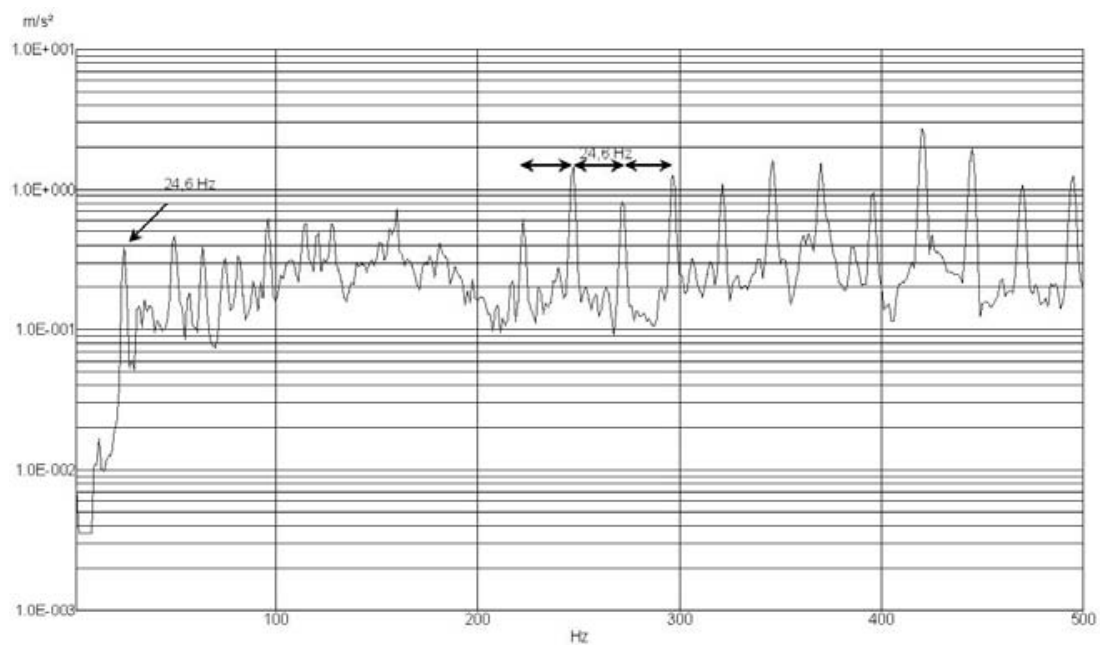

Fig. 20. Point de mesure P3 Rh - Spectre 0-500 Hz réducteur endommagé (échelle log).

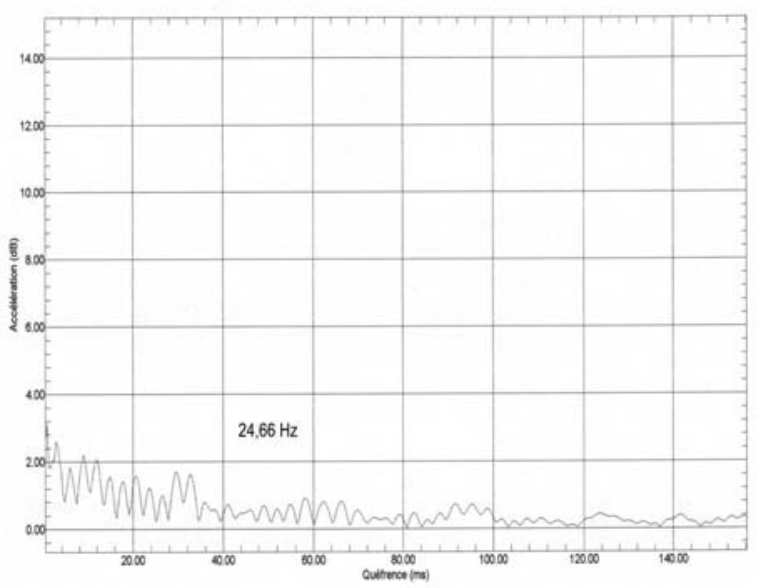

Fig. 21. Point de mesure P3 Rh - Cepstre sur $1000 \mathrm{~Hz}$ réducteur sain.

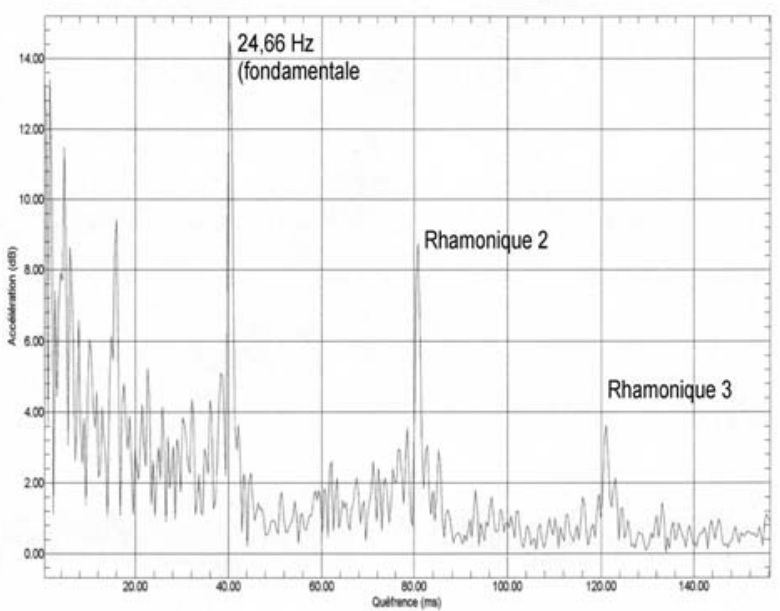

Fig. 22. Point de mesure P3 Rh - Cepstre sur $1000 \mathrm{~Hz}$ réducteur endommagé. 


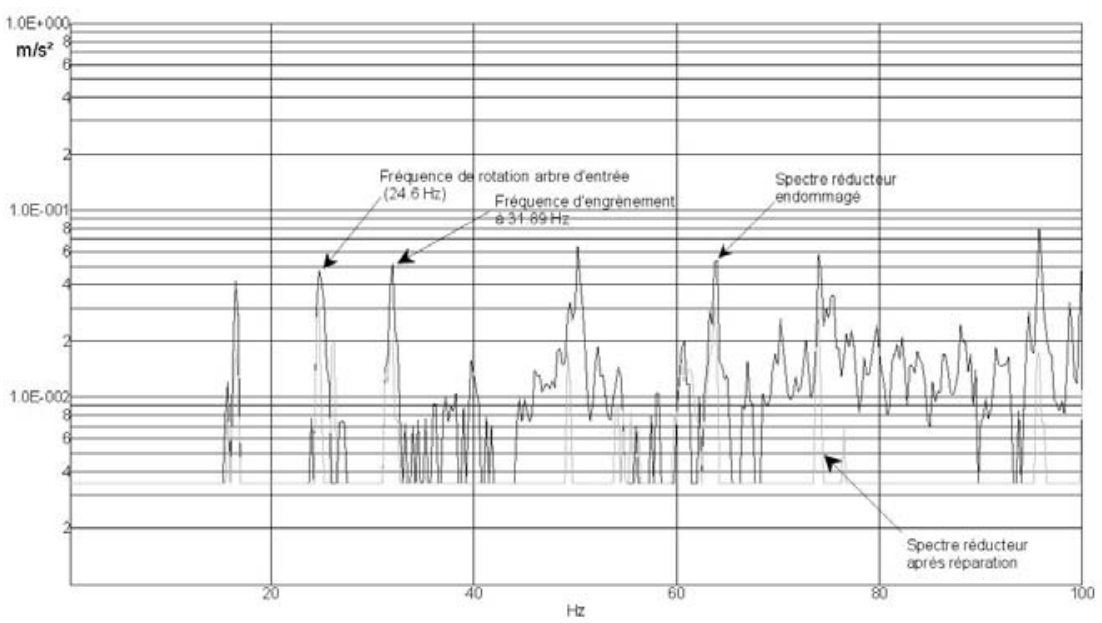

Fig. 23. Point de mesure P5 Rv - Spectre 0-100 Hz avec et sans défaut (échelle log).

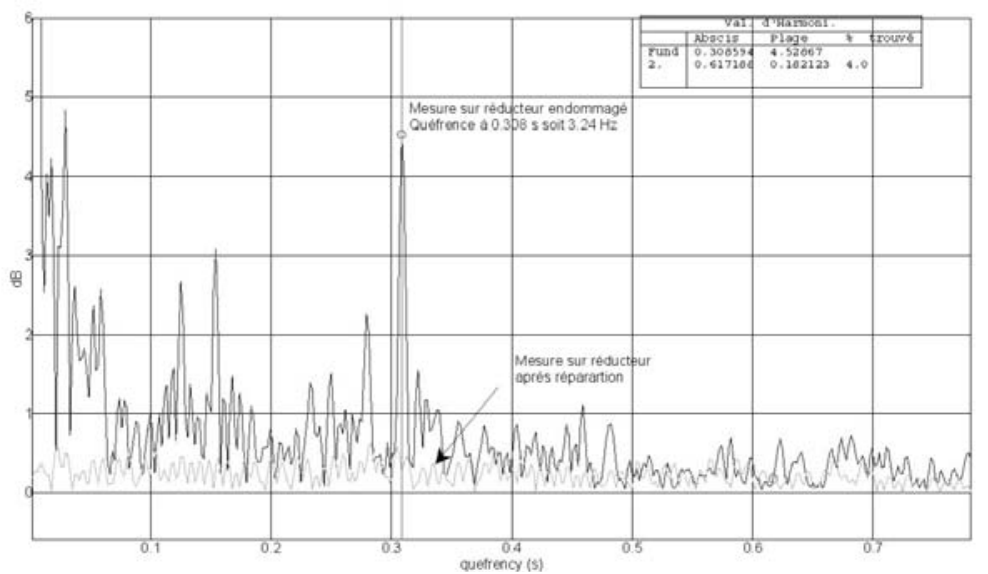

Fig. 24. Point de mesure P5 Rv - Cepstre sur $200 \mathrm{~Hz}$ avec et sans défaut.

de sortie $(0,52 \mathrm{~Hz})$. Un diagnostic sur l'état des engrenages $10 / 61$ dents et 58/58 dents dans ces conditions est impossible.

Le cepstre de la figure 24, à la différence du spectre, met en évidence un peigne de raie à la quéfrence de 0,308 s. Cette périodicité correspond à la fréquence de rotation de l'arbre intermédiaire du réducteur $(3,24 \mathrm{~Hz})$.

De même, le cepstre réalisé en très basse fréquence sur le point $\mathrm{P} 4$ en position axiale (Fig. 25) met en évidence un peigne de raies associé à la quéfrence de 1,91 s $(0,52 \mathrm{~Hz})$.

À partir de ces deux cepstres, nous pouvons en déduire qu'il existe des chocs périodiques associés à deux éléments cinématiques différents : l'arbre intermédiaire et l'arbre de sortie.

En outre, le fait de visualiser le peigne de raie associé à la quéfrence de 1,91 s uniquement dans la direction axiale sur le point $\mathrm{P} 4 \mathrm{Ax}$ laisse présager d'un mauvais guidage du pignon sur son arbre (frottement une fois par tour) plutôt que d'un défaut localisé sur une dent (choc à l'engrènement d'une dent saine avec la dent endommagée).
L'amplitude de la quéfrence fondamentale à $1,91 \mathrm{~Hz}$ des cepstres avant et pendant la détérioration a été multipliée par 3. Ceci nous incite à penser que le recours à des gabarits de contrôle sur la quéfrence fondamentale peut être tout à fait intéressant pour détecter des défauts. La mise en œuvre de ces gabarits ne pose pas de difficultés particulières, cependant l'évaluation des seuils d'alerte et de danger est délicate.

\subsubsection{Validation du diagnostic réalisé à l'aide de l'analyse cepstrale}

Tout comme dans l'application $\mathrm{n}^{\circ} 1$, nos résultats ont conduit le responsable maintenance à programmer un arrêt de l'installation afin de vérifier l'état de la denture du réducteur.

Cela a permis de mettre en évidence un arrachement de plusieurs dents consécutives sur le pignon de l'arbre d'entrée (Fig. 27) ainsi qu'une rupture de la liaison entre le pignon 61 dents et son alésage (Fig. 28). C'est d'ailleurs cette rupture qui est à l'origine de la détérioration de la denture des pignons 15, 116, 10 et 61 dents (voir Fig. 17). 


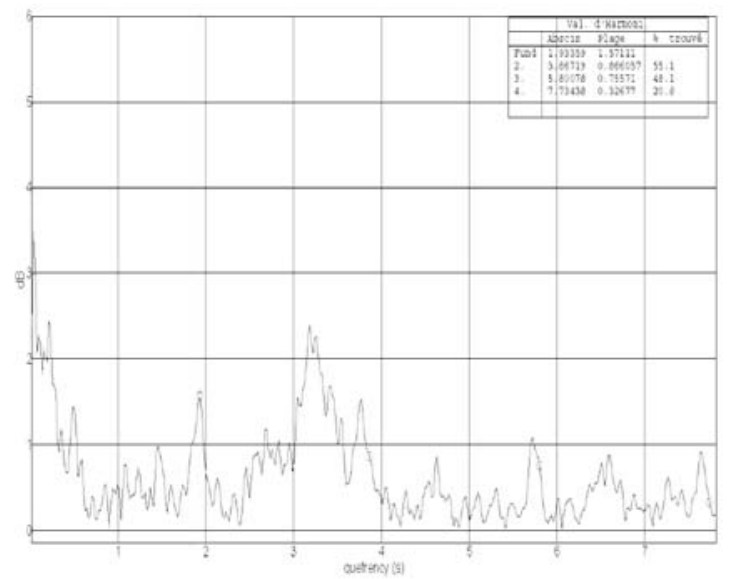

Fig. 25. Point de mesure P4 Ax - Cepstre sur $20 \mathrm{~Hz}$ réducteur sain.

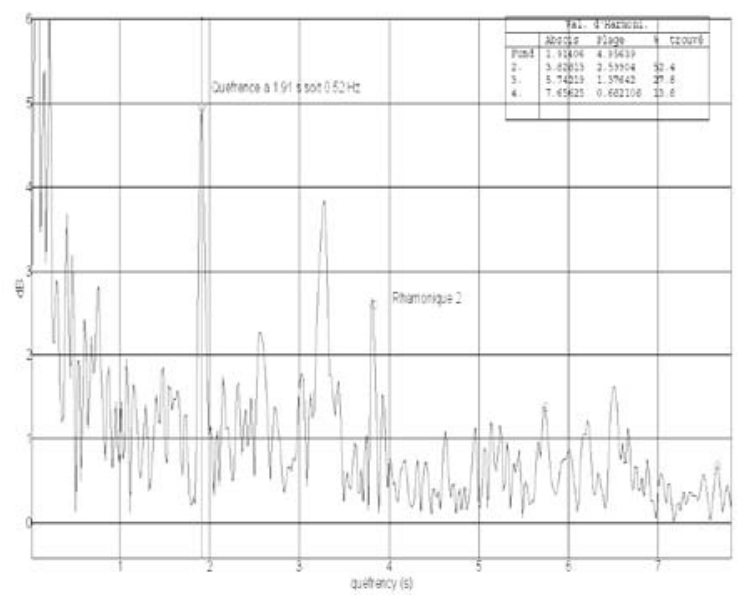

Fig. 26. Point de mesure $\mathrm{P} 4 \mathrm{Ax}$ - Cepstre sur $20 \mathrm{~Hz}$ réducteur endommagé.

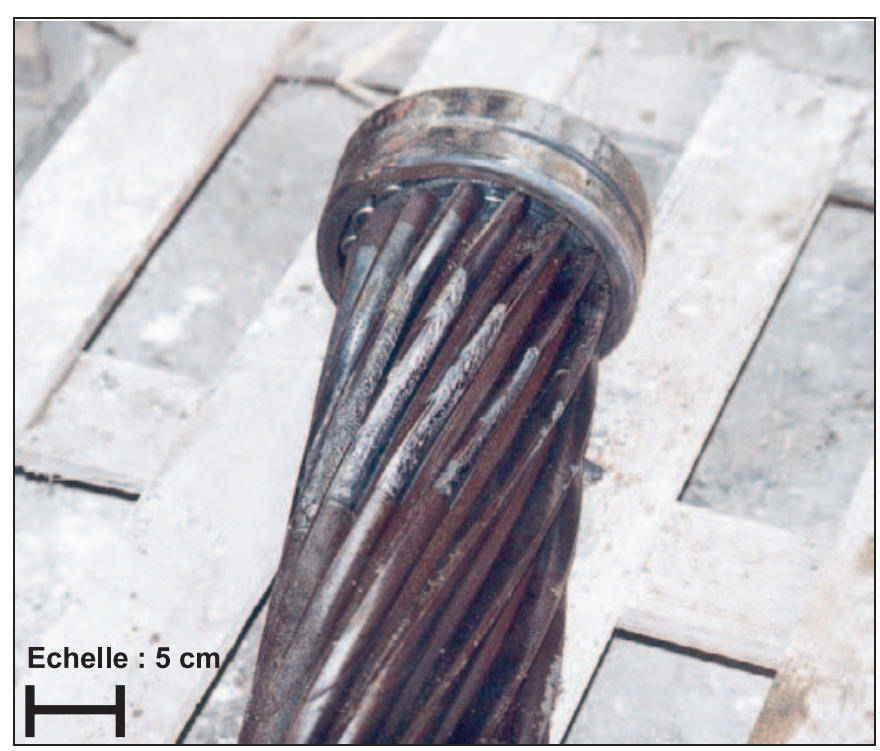

Fig. 27. Arrachement de plusieurs dents consécutives sur le pignon de l'arbre d'entrée du réducteur.

\section{Conclusions et perspectives}

Dans cette étude, nous avons évalué l'analyse cepstrale qui permet d'améliorer les performances de l'analyse spectrale dans la détection des défauts de type « choc » sur les réducteurs à engrenages. Cette évaluation a été menée en milieu industriel dans deux applications complémentaires :

- l'une sur un multiplicateur à vitesse élevée;

- l'autre sur un réducteur à vitesse lente.

Dans les deux cas, le cepstre s'est révélé particulièrement efficace pour détecter l'anomalie.

- Dans le premier, il met en évidence le défaut alors que le spectre ne fournit pas de symptôme.

- Dans le deuxième, il apporte, pour le premier train d'engrenage, une confirmation du diagnostic établi à partir de l'analyse spectrale et pour le deuxième train d'engrenage il a permis de détecter un défaut dont la fréquence était de $0,52 \mathrm{~Hz}$ (inexistante sur les spectres).

On montre également dans la seconde étude expérimentale qu'il est possible d'obtenir un diagnostic approfondi à partir de la comparaison de cepstres réalisés au même point mais dans deux directions de mesurage successives.

Par contre l'observation de l'évolution de l'amplitude de la «quéfrence» fondamentale pour caractériser le degré de sévérité de l'écaillage n'a pas été probante.

Dans le premier cas, un suivi sur plusieurs mois n'a pas permis de dégager une courbe de tendance régulière et fiable; on a seulement pu observer une augmentation systématique du cepstre.

Dans le deuxième cas, son évolution a été trop rapide à partir de la détection d'une anomalie pour pouvoir construire une loi d'évolution. Ce problème est lié à la nature du défaut « écaillage » qui, dans nos deux applications, évolue trop rapidement. Cependant la deuxième application nous laisse penser qu'il est possible de mettre en œuvre une maintenance prévisionnelle.

Le cepstre est calculé sur les analyseurs à partir du spectre; son implantation systématique sur les analyseurs ne pose pas de difficultés et les temps de calcul sont compatibles avec une utilisation industrielle. Il nécessite cependant un paramétrage précis pour obtenir la meilleure résolution possible. À cet effet, nous avons proposé, dans cette étude, des règles de paramétrage, liées aux caractéristiques des analyseurs, qui ont été efficaces dans les deux cas traités.

À partir de ces résultats, nos travaux s'orientent vers deux objectifs :

- Étudier l'influence de la gravité d'un écaillage sur le comportement dynamique des engrenages et donc sur l'évolution du cepstre, dans une perspective de maintenance prévisionnelle.

- Expérimenter le cepstre pour la détection précoce de certains défauts sur les paliers à roulements fortement chargé et fonctionnant à faible vitesse. 


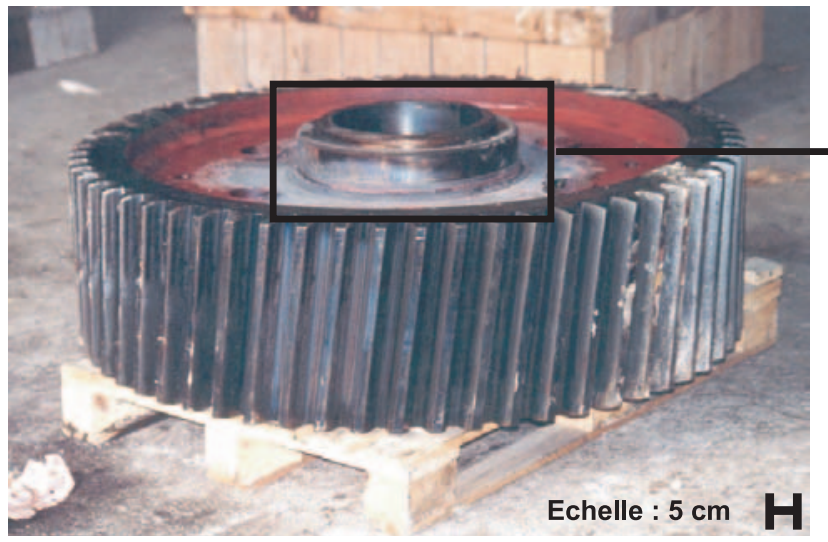

(a)

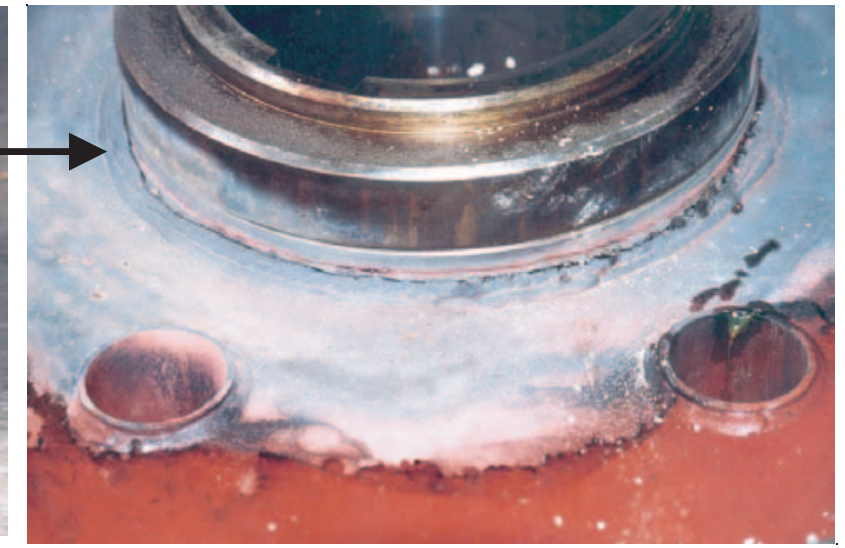

(b)

Fig. 28. (a) Photographie de la couronne 61 dents. (b) Mise en évidence de la rupture du cordon de soudure sur la couronne 61 dents.

\section{Références}

[1] G. Henriot, Traité théorique et pratique des engrenages, $7^{\mathrm{e}}$ édition, Dunod, 1999

[2] R.B. Randall, Cepstrum analysis and gearbox fault diagnostic, $2^{\mathrm{e}}$ édition, Publication Brüel \& Kjaer, 1977

[3] R.B. Randall, A new method of modeling gear faults, J. Mech. Design 104 (1982) 259-267

[4] C. Capdessus, M. Sidhamed, Analyse des vibrations d'un engrenage cepstre, corrélation, spectre, Revue Traitement du signal 8(5) (1992) 365-372

[5] M. El Badaoui, F. Guillet, N. Nejjar, Diagnostic d'un train d'engrenages par analyse cepstrale synchrone, $16^{\mathrm{e}}$ colloque
GRESTI sur le traitement du signal et des images, Grenoble 15-19 septembre 1997

[6] R.B. Randall, L'analyse cepstrale et le diagnostic des défauts dans les réducteurs/multiplicateurs, Notes d'applications Bruel \& Kjaer, 1980

[7] J.T. Kim, Cepstral analysis as a tool for robust processing, deverberation and detection of transients, Mechanical system and signal processing $\mathrm{n}^{\circ} 6$ (1992)

[8] R. Bigret, J.L. Feron, Diagnostic, maintenance, disponibilité des machines tournantes, collection technologie, Masson, 1994

[9] A. Boulanger, C. Pachaud, Diagnostic vibratoire en maintenance préventive, Dunod, 1998, pp. 241-259

Retrouvez nos articles sur le site : www.edpsciences.org/meca 